\title{
Research on Distributed Decentralized Management and Control Mode in Intelligent Networked Automobile Industry
}

\author{
Sun Xueyuan ${ }^{1, a}$, Shi Yuntao 2, , \\ ${ }^{1}$ Capital University of Economics and Business, Beijing, China 100070 \\ ${ }^{2}$ Northern China University of Technology, Beijing, China 100144 \\ asunxueyuan@cueb.edu.cn, bshiyuntao@ncut.edu.cn
}

\begin{abstract}
Keywords: Intelligent Networked Automobile, Emerging Industries, Distributed, Decentralized Management.
\end{abstract}

\begin{abstract}
Significant advancements in the intelligent vehicle and vehicle network technology have fostered an emerging industry- the intelligent network car industry. The distinctive characteristics of this industry have completely subverted the underlying laws of traditional industries and promoted innovation in enterprise management and business model remodeling. In this paper, the development of the intelligent network car industry is described in detail, and the characteristics of the industry are analyzed; an effective distributed decentralized management and control model is proposed, and the development direction of the industry and its prospect are predicted.

As an important product of the deep integration of informatization and industrialization, intelligent vehicles (unmanned autonomous vehicles) and Internet of vehicles have not only given rise to a new industry, but also greatly promoted enterprise management innovation and business model remodeling. The intelligent network automobile industry integrates burgeoning design, production, management, control and sales mode. Its subject, content and pace of innovation will bring unprecedented life experience to users. Intelligent vehicles and Internet of vehicles are a blend of many emerging technologies and intelligent management modes, such as real-time perception, Internet communication, autonomous driving, autonomous navigation, etc. ${ }^{[1]}$ As an emerging industry, the intelligent vehicle and Internet of vehicles industry has completely overturned the underlying laws that we used to rely on in manufacturing and management of automobiles. Therefore, it is of great practical significance to study in depth the development law of the intelligent netted vehicle industry, to constantly propose new thoughts of industry management and control, and to explore the distributed decentralized management and control mode to ensure the safety of Internet of vehicles information and the reliable operation of intelligent vehicles.
\end{abstract}

\section{Industry overview}

With economic advancement and scientific and technological revolution, the automobile industry has met significant opportunities for development and upgrading. Driven by "Internet + " and Intelligent Manufacturing", new-generation information technologies such as artificial intelligence, Internet and Big Data are deeply integrated with the automobile industry and have promoted the rapid development of the intelligent netted automobile industry. Even the capital and technology market of some famous Internet companies such as Google, Apple, Baidu, Alibaba, Tencent, and Huawei have entered the arena of competition in the intelligence automobile industry ${ }^{[2]}$.Not only do international automobile brands like Benz, BMW, Ford and Toyota joined the competition, Chinese local brands including BYD and Geely followed suit.

Although the United States, Japan and the European Union and other countries enjoyed a heads start in the intelligent network automobile industry, China has seen rapid development in this field in these years. Currently, the intelligent netted automobile industry is in the golden development period and at the demonstration and application stage in China. Smart cars are not only widely used and have great industrial potential, but also a key area for the country to grow and strengthen new industries, gain new competitive edges and shape new drives of development. The real-time online service of the Internet of vehicles has been widely used in various fields of economy and society, 
and has become an important symbol of national scientific research strength and an important direction of a new round of industrial revolution. The new intelligent transportation service model will build a significance milestone for improving traffic efficiency and safety monitoring level. According to the National Development and Reform Commission, Ministry of Science and Technology (MOST) and Ministry of Industry and Information Technology, the "Auto Industry Long-term Development planning' was jointly released, forecasting that by 2025 , a car driving auxiliary system, partially automatic driving, conditional functions such as automatic driving and fully automatic driving will be realized and the new car assembly rate will exceed $80 \%$.The car networking market size is 78.5 billion RMB in 2017, and is estimated to reach114.2 billion RMB in 2018 and its market scale will increase from 368.2 billion RMBin2022 ${ }^{[2]}$, which shows huge potential for growth. The market size development trend of China's intelligent network automobile industry is shown in Figure 1.

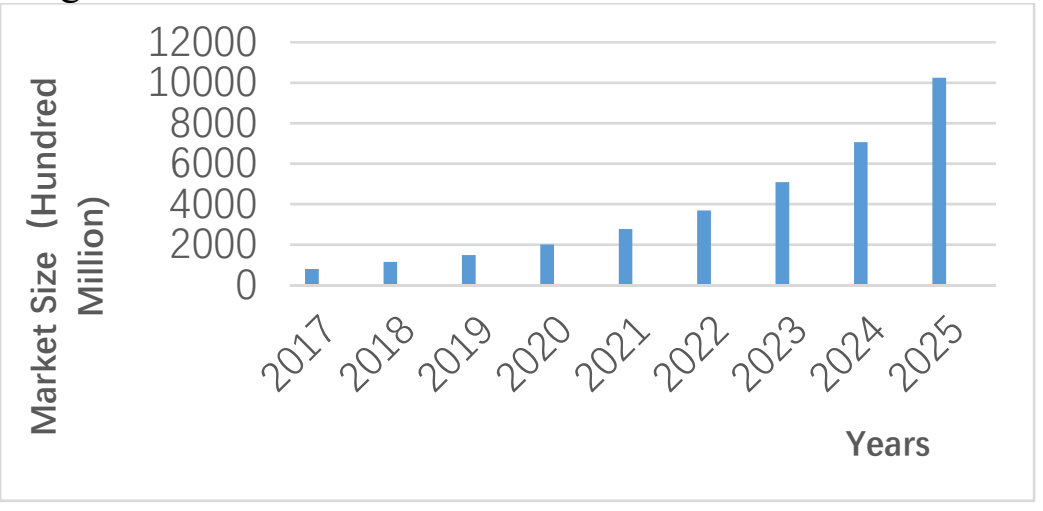

Figure 1 Forecast chart of market size of China's intelligent network automobile industry

\section{Characteristics of the industry}

The characteristics of the intelligent network link automobile industry are manifested in high integration of new generation information technology and intelligent control and intelligent management. Generally, it consists of two major industries: the single intelligent vehicles industry and the Internet of vehicles industry. It is a typical application and product of the era of traditional automobile and Internet + , and an emerging industry with the brightest future and market demand. In addition, to the traditional electromechanical structure and driving equipment, intelligent network connected vehicles also adds functions such as environment perception, information sharing, intelligent decision-making and autonomous control. Generally, it realizes the information connection and distributed management and control among vehicles, vehicle and management companies, and vehicle and traffic management departments. Through the Internet platform and network communication technology, it develops rapidly towards the direction of networking and intellectualization, and finally realizes the management and controlling objectives such as comfort, safety, efficiency and energy saving of intelligent networked vehicles. As shown in Figure 2, the industry of the intelligent network automobile industry has strong support from the capital chain and the service chain. It mainly includes three chains: production, service and capital. It involves not only traditional automobile manufacturers, $4 \mathrm{~S}$ stores and users, but also many service providers such as intelligent vehicle systems, Internet of vehicles, navigation and telecommunication providers ${ }^{[3]}$. At present, China has an edge in the fields of artificial intelligence, big data, Internet of things and $5 \mathrm{G}$ communication technologies. It has a complete supporting system for production, learning, research and application. National related management departments and production enterprises should promptly seize opportunities for development of the intelligent auto industry, strengthen the top-level design and standards, promote research and development of communication chips and control modules, and support automated driving technology and demonstration application. They should also push forward construction of car networking integrated data platforms and open interfaces, constantly explore new technology for the future of intelligent travel 
service, the new model, building security, intelligence, sharing systems of comprehensive transportation services, research and seek breakthroughs in key technologies such as on-board chips, wireless communications, autonomous driving, intelligent perception and operating systems to make the fullest of China's advantages in the field of new energy vehicle manufacturing and push the intelligent netted vehicle industry to a commanding height in the world.

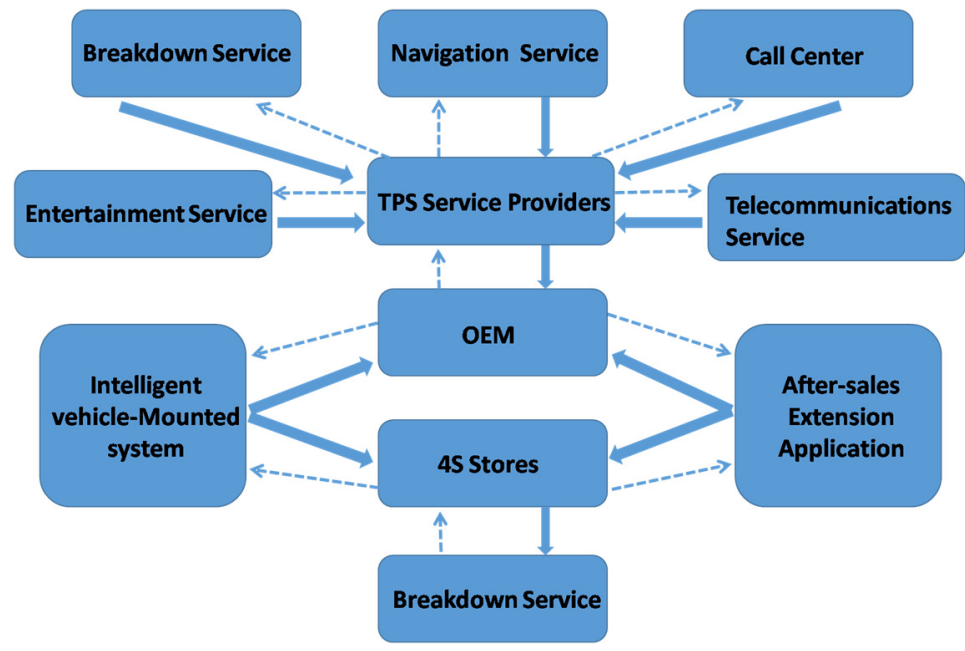

Figure 2 Schematic diagram of the development of intelligent network automobile industry chain

\section{Distributed decentralized management and control mode}

Distributed decentralized control is an effective means to manage and control complex systems. In general, the management and control of intelligent networked automobile industry is realized based on the Internet of vehicles and cloud platform through big data real-time processing. The so-called distributed decentralized management and control is the combination of networked decentralized control and centralized management ${ }^{[6]}$. Generally, it is composed of two layers of management and control: the first is the decentralized control layer which is mainly composed of information perception, real-time communication, optimal control, autonomous driving and safety prevention, with obvious real-time and safety characteristics. The centralized management is based on information transmission, data processing, path navigation and optimization decision-making, which has the obvious characteristics of high efficiency and reliability. The other is the decentralized control layer, each made vehicles as an independent agent, by decentralized control can be achieved when the system is a technical fault or running safety, each smart cars still have independent real-time control and security ability, even if the individual intelligent car or parts of technical failure also not affect network out of control and lead to paralysis of the whole system, greatly improved the overall security of the network system, can reduce the risk control to the greatest extent. The centralized management of the system through the big data platform of the Internet of vehicles can realize the sharing of road conditions and information resources, make path planning and navigation more reasonable, and make management decisions and optimization services more efficient. With rapid development of the intelligent vehicle and Internet of vehicles technology, and driven by the new generation of information technology, the intelligent Internet vehicle industry is developing towards the direction of diversification, networking, openness, centralized management and decentralized control. Through the smart grid of the general assembly data platform, the distributed decentralized management and control mode can realize more efficient traffic administration of different vehicles and different areas, in which t data storage, data transmission, data mining and data analysis and processing functions, such as cars and people, vehicles and road, car and direct connectivity of car information can be realized to promote efficient control and supervision of traffic administration of unmanned vehicles. At the same time, more and more professional multimedia and mobile Internet application services should be provided to allow car users to enjoy more comfortable driving experience. The distributed decentralized management and control mode of the intelligent network vehicle is shown in Figure 3. 


\section{Vehicle Network Big Data Centralized Management Platform}

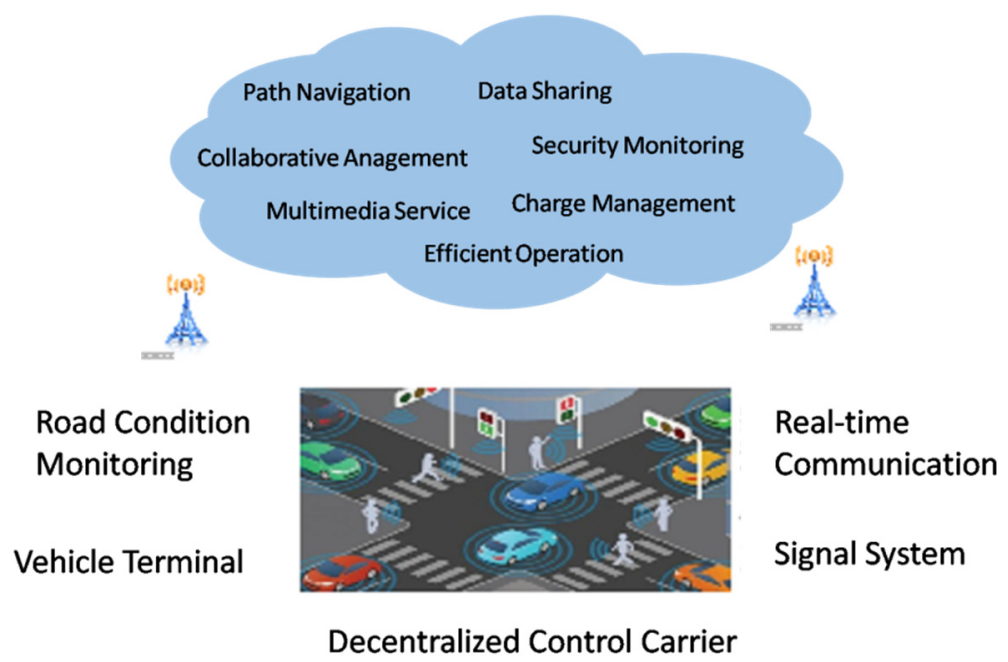

Figure 3 Distributed decentralized management and control mode

The distributed control system of intelligent cars is a multidisciplinary, interdisciplinary and comprehensive method. To study the new thinking of management and control integration by using the rolling optimization and integrated research method for transportation system management and control scheme of the online simulation, evaluation and optimization, is of vital significance. To realize intelligent Shared league of monomer is the key link in the car network. Vehicle information such as position, velocity and road conditions are network transmission to the intelligent traffic management center by car cloud platforms, and then by the information technology of data analysis and processing, and the location of different vehicles, the best route and energy saving operation mode is calculated. Generally, intelligent vehicles and Internet of Vehicles can fully realize intelligent traffic information service and distributed decentralized control of intelligent vehicles after they go through three stages of information interaction, coordination and perception, and network decision-making.

\section{Development direction}

To promote the development of the intelligent automobile industry, state and local governments have introduced related support policy and standard specification, paid attention to the bottleneck problem of management, strengthened the innovation mode, the top design, traffic accident analysis and positive research of mechanisms. It will intensify research of key technology of common, promote industrialization projects and major industrial base platform construction to fall to the ground as soon as possible, explore the implementation of traffic data open sharing, improve the protection of intellectual property rights, actively participate in international standards, strengthen international exchanges and cooperation, promote the international influence of the industry.

In the future, the development of the intelligent network auto industry should focus on the following six aspects ${ }^{[5]}$ :

First, it will continue to innovate systems and mechanisms, accelerate the technological upgrading and transformation of traditional automobiles, change traditional driving behaviors and gradually accept the concept of unmanned driving.

Second, it will promote actively the large-scale manufacturing and technology industrialization of the core components of intelligent network vehicles, transform the vehicle manufacturing system of driverless cars, accept personalized customization, and build a networked collaborative intelligent manufacturing industry chain.

Third, it will speed up the development of intelligent road network technology with vehicle-road collaboration as the core, carry out intelligent road network reconstruction and satellite ground station construction, and improve the overall perception of the road network. 
Fourth, the smart travel management system and service mode should be established as soon as possible to realize the optimization of traffic signals, traffic organization and traffic guidance based on the big data cloud platform.

Then, efforts will be made to improve the road intelligent network communication infrastructure, and develop 5G-basedchips for Internet of vehicles, communication base stations, vehicle-mounted terminals and other key networking equipment.

Lastly, we will vigorously develop the high-precision digital map and Beidou Navigation industry, and support the development of the self-organizing, self-learning and self-adaptive capabilities of the internet-connected vehicles.

\section{Application Prospect}

The intelligent network automotive industry has become the hotspot in a new round of global industrial competition. The governments, industry professionals and the scientific and technological circles have accelerated the layout in this field, and take the lead in the demonstration and application of private cars, freight and rental in airports, docks, stations and other public places, and then gradually expand to the field of family and personal applications. Although China started late in the field of intelligent network vehicles, the development speed is beyond the world's expectation. Beijing has made it clear that by 2022, a complete technical system and application service standards for intelligent networkers will be basically formed. The demonstration operation area will exceed 500 square kilometers, and the industrial scale will reach 100 billion RMB ${ }^{[5]}$. Huawei has also officially entered the field of Internet of vehicles, and has successively cooperated with Audi, Volkswagen, Toyota, Dongfeng, and other vehicle manufacturers in the fields of intelligent vehicles and Internet of vehicles. Baidu's research and development of car networking open platform (apollo) has been designated by the government as an innovation center of the new generation of artificial intelligence, and with major car manufacturers like BMW, Mercedes-Benzes, Ford, Hyundai, Honda, as well as new-energy brands like BYD, unmanned vehicles they developed jointly have been on the road to the Xiongan and Hong Kong-Zhuhai-Macao bridge..Alibaba also recently announced the development of a new Internet of things operating system, which will allow drivers to make reservations and conduct online settlement via automap through its Alipay online platform. Tencent is developing a car networking control platform based on QQ and WeChat. Its unique product content and services positioning attracted many heavyweight partners, including Volkswagen, Ford, gac, Changan, Geely, Dongfeng, and BYD.The platform, it has facilitated many people's travel and realized location sharing and real-time intercom driving, opening a new era of the comprehensive intelligent cars. The development and technology promotion and application of intelligent network vehicle industry in the next five years are shown in Figure 4

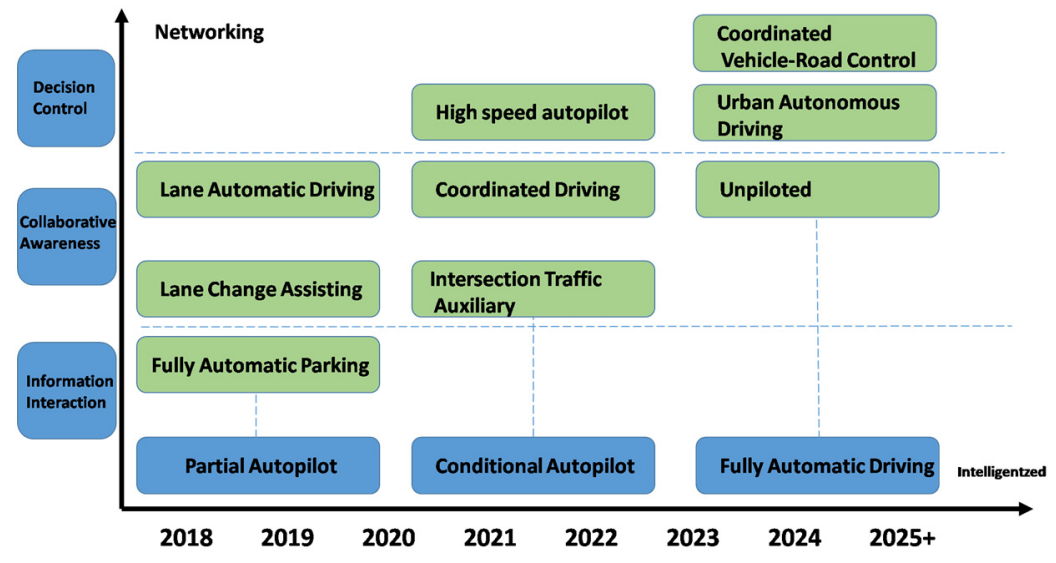

Figure 4 Network car industry technology development trend chart

Some autonomous driving can be achieved by 2020, including fully automatic parking, lane changing assistance and automatic driving in general lanes. Conditional autonomous driving can be 
realized in 2022, including automatic crossing, coordinated queue driving and high-speed autonomous driving. After 2024, fully automatic driving will be realized with more advanced functions such as unmanned automatic driving and coordinated optimization control of vehicles and roads in the urban area.

\section{References}

[1] Li Keqiang et al, Status and trends in the development of intelligent networked vehicles (ICV) technologies, Journal of Automotive Safety and Energy, 2017(8).

[2] Sunning et al, Analysis of the development of intelligent network-connected automobile industry, Report on the Development of China's Intelligent Net Link Automotive Industry,2018(9).

[3] Document of Ministry of Industry and Information Technology, Action Plan for the Development of the Industry In the Networking (Smart Networked Vehicle),2018(12).

[4] Li Yuxian et al, Summary of the development of intelligent net-linked automotive safety, China Automotive Safety Development Report,2018(9).

[5] Chen Chunmei et al, Policy system of smart net-linked vehicle support and industrial development proposal, Report on the Development of China's New Energy Vehicle Industry,2018(8).

[6] Sun Dehui, Shi Yuntao et al, Networked control system, Defense Industry Press, 2008(5).

[7] MiadFaezipour, MehrdadNourani, Adnan Saeed, StateeshAddepalli, Progress and challenges in intelligent vehicle area networks, Communications of the ACM,2012.

[8] Paniati J F, Vehicle infrastructure integration, VII Public Meeting,2015.

[9] ZhigangXu, Mingliang Wang, Xiangmo Zhao, et al. PaTAVTT: A Hardware in the loop Scaled Platform ;

[10]Zhao Fuquan, Kuang Xu, Liu Zongwei, Research on the industrial upgrade of automotive industry for intelligent net-linked cars: based on the perspective of value chain, Scientific and Technological Progress and Countermeasures, 2016(17).

[11]Liu Tianyang, Yu Zhuoping, Xiong Lu, Zhang Peizhi, the development status and construction of intelligent net-linked automobile test site

[12] Make recommendations, Automotive Technology,2017(01).

[13]Kong Chuiying, Ma Enhai, Men Feng, Research on the development path and mechanism of intelligent network-connected automobile, Automotive Industry Research, 2016(11).

[14]WANG Jianqiang, LI Shengbo, HUANG Xiaoyu, et al. Driving simulation platform applied to develop drivingassistance systems, IET Intell TranspSyst,2010, 4(2):121-127.

[15]Erhan D, Szegedy C, Toshev A, et al, Scalable objectdetection using deep neural networks,IEEE ConfComputer Vision and Pattern Recognition (CVPR), 2014:2147-2154.

[16]U.S. Department of Transportation, Status of the dedicated short-range communications technology and applications report to congress, 2015.

[17]Zhu Tongyu, Wang Jiachuan, Chen Zhihong, Early exploration of car networking technology, Highway Traffic Technology: Application Technology Edition,2011(5), pp.266-268.

[18]Huang Haikun, Deng Jiajia, Internet of things gateway technology and applications, Telecommunications Science,2010(4), pp.20-24. 